\title{
The relative importance of prey-borne and predator-borne chemical cues for inducible antipredator responses in tadpoles
}

\author{
Attila Hettyey ${ }^{1,2,3} \cdot$ Zoltán Tóth $^{1,3} \cdot$ Kerstin E. Thonhauser $^{1} \cdot$ Joachim G. Frommen $^{1,4}$. \\ Dustin J. Penn ${ }^{1} \cdot$ Josh Van Buskirk ${ }^{2}$
}

Received: 7 June 2014 / Accepted: 21 June 2015 / Published online: 11 July 2015

(C) Springer-Verlag Berlin Heidelberg 2015

\begin{abstract}
Chemical cues that evoke anti-predator developmental changes have received considerable attention, but it is not known to what extent prey use information from the smell of predators and from cues released through digestion. We conducted an experiment to determine the importance of various types of cues for the adjustment of anti-predator defences. We exposed tadpoles (common frog, Rana temporaria) to water originating from predators (caged dragonfly larvae, Aeshna cyanea) that were fed different types and quantities of prey outside of tadpolerearing containers. Variation among treatments in the magnitude of morphological and behavioural responses was highly consistent. Our results demonstrate that tadpoles can assess the threat posed by predators through digestionreleased, prey-borne cues and continually released predator-borne cues. These cues may play an important role in
\end{abstract}

Communicated by Ross Andrew Alford.

Electronic supplementary material The online version of this article (doi:10.1007/s00442-015-3382-7) contains supplementary material, which is available to authorized users.

Attila Hettyey

hettyey.attila@agrar.mta.hu

1 Department of Integrative Biology and Evolution, Konrad Lorenz Institute of Ethology, University of Veterinary Medicine Vienna, Vienna, Austria

2 Institute of Evolutionary Biology and Environmental Studies, University of Zurich, Zurich, Switzerland

3 Lendület Evolutionary Ecology Research Group, Plant Protection Institute, Centre for Agricultural Research, Hungarian Academy of Sciences, Budapest, Hungary

4 Department of Behavioural Ecology, Institute for Ecology and Evolution, University of Bern, Hinterkappelen, Switzerland the fine-tuning of anti-predator responses and significantly affect the outcome of interactions between predators and prey in aquatic ecosystems. There has been much confusion regards terminology used in the literature, and therefore we also propose a more precise and consistent binomial nomenclature based on the timing of chemical cue release (stress-, attack-, capture-, digestion- or continually released cues) and the origin of cues (prey-borne or predator-borne cues). We hope that this new nomenclature will improve comparisons among studies on this topic.

Keywords Alarm signal · Inducible defence ·

Kairomone $\cdot$ Phenotypic plasticity $\cdot$ Predator labelling

\section{Introduction}

Prey have evolved a variety of mechanisms that lessen the threat of predation, including behavioural, physiological and morphological responses. These responses are not necessarily present at all times, but can be induced by signals indicating predation risk. The expression of inducible defences is expected to be optimally adjusted, within the limits of plasticity, such that protection is maximized and costs are minimized (Harvell 1990; DeWitt et al. 1998; Tollrian and Harvell 1999). Adaptively adjusting inducible defences requires that prey are able to detect reliable cues regarding the type, abundance and dangerousness of predators present in the environment (Moran 1992). Different kinds of cues may be favoured in different ecological contexts, but in aquatic environments-and especially in turbid waters-chemical cues are considered the most important sensory modality for detecting predators (Kats and Dill 1998; Tollrian and Harvell 1999; Brönmark and Hansson 2000). 
Table 1 A tabulated summary of the suggested terminology and classification of chemical cues of predation threat

\begin{tabular}{llll}
\hline Timing of release & Popular term & Suggested term & Constituents \\
\hline \multirow{2}{*}{ Pre-consumption } & Indirect cues & Prey-borne cues & \\
& No-cost disturbance signals & Stress-released cues & General prey metabolites \\
& Alarm pheromones & Attack-released cues & Alarm pheromones \\
& Damage-released cues & Capture-released cues & Alarm pheromones, tissue fragments \\
Post-consumption & Digestion-released cues & Digestion-released cues & Constituents of digested prey \\
& Direct cues & Predator-borne cues & \\
Pre-consumption & - & Capture-released cues & Saliva \\
Post-consumption & Kairomones/digestion-released cues & Digestion-released cues & Digestive fluids, digestive tract tissue, gut flora \\
Continuously & Kairomones & Continually released cues & Chemicals and tissue fragments from integument \\
\hline
\end{tabular}

Many studies have demonstrated the induction of antipredator defences mediated through chemical cues, but drawing general conclusions about the underlying mechanisms has been hampered by ambiguities and differences in terminology and definitions (see Appendix, Box 1). Therefore, we suggest a new terminology and a classification of terms regarding chemosensory-mediated predator detection (Table 1), which we hope will help clarify our study and future studies as well. Henceforth, we use this new terminology. We collectively refer to stress-, attack- and capturereleased prey-borne cues as pre-consumption prey-borne cues throughout the text because the experimental design does not allow us to differentiate among their effects.

Numerous studies demonstrate the role of pre-consumption prey-borne cues in the induction of antipredator responses (for a review see Chivers and Smith 1998), but similarly comprehensive and convincing studies of continually released predator-borne cues and digestion-released prey- or predator-borne cues are scarce. One recurring problem is the uncertainty about whether prey-borne cues are present. Studies designed to investigate effects of predator-borne cues often do not report how long predators were deprived of food before exposing them to focal prey. Even if the duration of food restriction is known, it is not always clear that prey-borne cues are completely absent. The rate of degradation of pre-consumption prey-borne cues has been measured (Peacor 2006; Ferrari et al. 2008; Van Buskirk et al. 2014), but predators may defecate long after they consumed prey and digestion-released prey-borne cues may therefore persist. Observed prey responses may therefore not be attributed solely to continually released predator-borne cues (but see Petranka and Hayes 1998; Schoeppner and Relyea 2009). Also, the results of studies that investigate whether prey exploit information contained in digestion-released cues are inconclusive (e.g. Laurila et al. 1997, 1998; Schoeppner and Relyea 2005, 2009; Richardson 2006; Ferrari et al. 2007; Ferland-Raymond et al. 2010). Differences among treatments cannot unambiguously be assigned to effects of digestion-released cues, because pre-consumption prey-borne cues or continually released predator-borne cues are not always eliminated, or a synergistic effect between these two cannot be excluded.

Clarifying the origin of chemical cues is critical for understanding the proximate mechanisms through which aquatic prey detect predators and express antipredator defences. It has been argued that prey use predator-borne cues to adjust the type of response, and prey-borne cues to adjust the intensity of response (Kishida and Nishimura 2005; Teplitsky et al. 2005; Wilson et al. 2005; Schoeppner and Relyea 2008; Hettyey et al. 2010). Also, while predator-borne and prey-borne cues can induce behavioural responses in isolation in some species (Petranka and Hayes 1998; Fraker et al. 2009), both types of chemical cues may be necessary for developing the full suite and magnitude of induced defences (Van Buskirk and Arioli 2002; Schoeppner and Relyea 2005, 2009; Richardson 2006; Hettyey et al. 2010).

In theory, predator-borne cues and digestion-released cues could provide prey with very specific information on the abundance, location and recent feeding habits of the predators in their environment, while pre-consumption prey-borne cues could provide more general information about the whereabouts and overall feeding activity of predators. Also, prey that rely solely on pre-consumption cues would not detect predators that have not fed recently. Finally, we expect predator-borne and digestion-released cues to be used by prey when adjusting their phenotypic responses to predation threat because different responses vary in their effectiveness against different types of predators, and predators may differ in their activity profile and in their food and microhabitat preferences.

This study was designed to disentangle effects of continually released predator-borne cues and digestion-released prey- or predator-borne cues on the antipredator responses of prey. We used combinations of different cue types, which allowed us to evaluate the relative importance of the cues and estimate the suite of cues necessary for the induction of the full intensity of inducible defences. We used tadpoles of 
the common frog (Rana temporaria), which are known to adjust their behaviour and morphology in response to predators (e.g. Laurila et al. 1997; Van Buskirk 2001; Teplitsky and Laurila 2007). By using predator-naive tadpoles, we excluded any confounding effects of learning (e.g. Gonzalo et al. 2007; Fraker 2009; Chivers and Ferrari 2013). We addressed the following main hypotheses:

1. Continually released predator-borne cues and digestion-released cues interact with pre-consumption cues and with each other in eliciting a response.

2. Effects of digestion-released cues are graded according to the phylogenetic distance between focal tadpoles and prey.

3. Varying quantities of digestion-released cues result in graded responses in tadpoles.

\section{Materials and methods}

\section{Experimental design}

We performed an outdoor mesocosm experiment in which $R$. temporaria tadpoles were exposed to ten treatments, each replicated ten times in a randomized spatial block design. A high level of replication was necessary to deliver the power to evaluate hypotheses where previous studies failed to provide decisive answers [e.g. effect of continually released predator-borne cues in isolation (Schoeppner and Relyea 2009)]. The ten treatments exposed focal tadpoles to chemical cues of different sources and kinds (Table 2):
- A no-predator control provided baseline data for the description of the predator-naive tadpole phenotype (T1).

- Predators fed with live conspecific prey provided all types of chemical cues (T2).

- Homogenized tadpoles in the absence of predators exposed focal tadpoles to pre-consumption prey-borne cues (T3).

- A starved predator allowed only continually released predator-borne cues (T4).

- Homogenized tadpoles together with a starved predator provided a combination of pre-consumption prey-borne cues and continually released predator-borne cues while excluding digestion-released cues (T5).

- Predators fed with Chironomus midge larvae, Bufo bufo tadpoles, Rana arvalis tadpoles, or Rana temporaria tadpoles, respectively, and subsequently washed to remove pre-consumption prey-borne cues, so that a combination of continually released predator-borne cues and digestion-released cues was present; the digestion-released cues originated from four prey taxa that differed in their phylogenetic relatedness to the focal tadpoles (T6-T9).

- Predators fed twice as much conspecific prey and subsequently washed, to provide elevated levels of digestionreleased cues (T10).

Table 2 summarizes which kinds of cues were present in each treatment.

The experimental design allowed us to make three kinds of comparisons. (a) We tested whether cue type affected antipredator responses by comparing T1-T5 and T9. These
Table 2 A list of procedures and the types of cues present in the ten treatments [predator $(P)$, only handling of an empty cage (no $P)$, Rana temporaria tadpoles (Rt), homogenized Rana temporaria tadpoles (Rt mix), chironomid larvae (Ch), Bufo bufo tadpoles $(B b)$, Rana arvalis tadpoles $(R a)$, double amount $(2 \times)$, predator washed three times after feeding (wash)]

\begin{tabular}{|c|c|c|c|c|c|}
\hline \multirow[t]{2}{*}{ Treatment code } & \multirow[t]{2}{*}{ Procedure } & \multicolumn{2}{|l|}{ Prey-borne cues } & \multicolumn{2}{|l|}{ Predator-borne cues } \\
\hline & & Pre-consumption & Digestion-released & Digestion-released & Continually released \\
\hline $\mathrm{T} 1$ & No $P$ & - & - & - & - \\
\hline $\mathrm{T} 2$ & P fed Rt & + & + & + & + \\
\hline $\mathrm{T} 3$ & Rt mix & + & - & - & - \\
\hline $\mathrm{T} 4$ & Starved P & - & - & - & + \\
\hline T5 & Starved P + Rt mix & + & - & - & + \\
\hline T6 & $\mathrm{P}$ fed $\mathrm{Ch}+$ wash & - & + & + & + \\
\hline $\mathrm{T} 7$ & $\mathrm{P}$ fed $\mathrm{Bb}+$ wash & - & + & + & + \\
\hline $\mathrm{T} 8$ & $\mathrm{P}$ fed $\mathrm{Ra}+$ wash & - & + & + & + \\
\hline T9 & P fed Rt + wash & - & + & + & + \\
\hline $\mathrm{T} 10$ & $\mathrm{P}$ fed $2 \times \mathrm{Rt}+$ wash & - & + & + & + \\
\hline
\end{tabular}

This design does not distinguish between various types of pre-consumption prey-borne cues, but rather focuses on digestion-released cues of both origins and on continually released predator-borne cues. We do not list pre-consumption predator-borne cues (present only in T2) because we know very little about them and the design does not support conclusions regarding their importance 
analyses investigated whether prey-borne and predatorborne cues interacted with each other and with pre-consumption cues in determining the strength of responses. These comparisons were also suitable for assessing if all types of cues were necessary to mount the full intensity of inducible defences. (b) We assessed the hypothesis that prey type matters for inducible defences by comparing $\mathrm{T} 4$ and T6-T9. These analyses tested whether effects of digestionreleased cues are graded according to the phylogenetic distance between focal species and the prey consumed by the predator, as had been observed for pre-consumption cues (Laurila et al. 1997, 1998; Schoeppner and Relyea 2005; Fraker 2009). Finally, (c) we investigated the importance of prey quantity based on $\mathrm{T} 4, \mathrm{~T} 9$, and $\mathrm{T} 10$. These comparisons tested whether varying quantities of digestion-released cues resulted in graded responses in focal tadpoles, as they do when all cues are available (Van Buskirk and Arioli 2002; Ferrari et al. 2005; Fraker 2008; McCoy et al. 2012). A positive result would provide another line of evidence for sensitivity to digestion-released cues by tadpoles.

\section{Experimental procedures}

The experiment was conducted in rectangular plastic mesocosms $\left(29 \mathrm{~L}, 0.18 \mathrm{~m}^{2}\right)$, covered with mosquito netting and placed outdoors at the Konrad Lorenz Institute of Ethology, Vienna. We used relatively small rearing containers to be able to obtain adequate sample sizes and statistical power (see above). Mesocosms were filled with tap water 2 weeks before the start of the experiment. Two days later we stocked mesocosms with $15 \mathrm{~g}$ of dried leaves (Fagus sylvatica) to provide shelter and nutrients for tadpoles, and added to each mesocosm $1 \mathrm{~L}$ of water containing phytoand zooplankton from a nearby pond to enhance algal growth and maintain water quality. Each mesocosm was fitted with a predator cage made of opaque plastic tube; a double net bottom allowed free exchange of chemical cues while preventing predators from injuring focal tadpoles. Visual and tactile cues may also play a role in predator detection (Stauffer and Semlitsch 1993; Hettyey et al. 2012), but chemical cues seem to be the most important for tadpoles, and strong antipredator responses have been reported when only chemical cues were available to focal individuals (Stauffer and Semlitsch 1993; Ferland-Raymond et al. 2010; Winkler and Van Buskirk 2012).

The experimental animals were hatched from eggs deposited in captivity by ten pairs of adult $R$. temporaria collected at a pond near Vienna $\left(48^{\circ} 13^{\prime} \mathrm{N}, 16^{\circ} 17^{\prime} \mathrm{E}\right)$. Clutches were reared separately in containers placed outdoors until the experiment began, and tadpoles were completely naive to predators. Predators in this study were larvae of the dragonfly Aeshna cyanea, because these are abundant and important predators of anuran tadpoles in central European wetlands (e.g. Van Buskirk 2009; Hettyey et al. 2011). The A. cyanea dragonfly larvae (instars F-1 and F-2) came from a pond in Hungary $\left(47^{\circ} 44^{\prime} \mathrm{N}\right.$, $\left.19^{\circ} 01^{\prime} \mathrm{E}\right)$ and food for the dragonflies came from ponds in Styria $\left(46^{\circ} 46^{\prime} \mathrm{N}, 15^{\circ} 39^{\prime} \mathrm{E} ;\right.$ R. arvalis $)$ and Vienna $\left(48^{\circ} 12^{\prime} \mathrm{N}\right.$, $16^{\circ} 15^{\prime} \mathrm{E}$; B. bufo), or from a local pet shop (live chironomids). The predators were kept individually in 0.3-1 cups and fed on chironomid larvae and $R$. temporaria tadpoles until 3 days before the start of the experiment. In a preliminary study, we confirmed that $A$. cyanea larvae do not defecate after being deprived of food for 3 days. It has been claimed that not only defecation may generate digestionreleased prey-borne cues (Brown et al. 1995), but cues that label the predators as dangerous are known to degrade within 48 h or less (Peacor 2006; Ferrari et al. 2008; Van Buskirk et al. 2014).

Predators were fed every day, except when we performed behavioural observations (see below). At feeding events, we brought predators to the laboratory and fed them in 100-ml cups with $25 \mathrm{mg}$ of the appropriate prey. The two ground-tadpole treatments were prepared $1 \mathrm{~h}$ after the predators were fed, by placing $600 \mathrm{mg}$ of $R$. temporaria tadpoles into $120 \mathrm{ml}$ of aged tap water and grinding them with a mixer. Tadpoles were dead within seconds of turning on the mixer, but we intentionally did not anaesthetize them to ensure that anaesthetics were not present and that tadpoles were not unconscious preceding death. Homogenized tadpoles have been used in similar studies as a source of pre-consumption prey-borne cues and are well known to induce clear responses in prey (e.g. Petranka and Hayes 1998; Schoeppner and Relyea 2005; Ferrari et al. 2008; but see Fraker et al. 2009). Feeding cups in T2 and T4 each received $5 \mathrm{ml}$ of this tadpole mixture. Two hours after the start of feeding, we removed any uneaten prey from the cups and washed predators in T6-T10 by pouring out and refilling feeding cups three times. In previous studies, preconsumption cues were reduced below detectability by washing predators once (LaFiandra and Babbitt 2004) or twice (Richardson 2006) or by changing the predator water after feeding and waiting for $24 \mathrm{~h}$ (Ferland-Raymond et al. 2010). Consequently, it seems likely that washing predators three times effectively excluded pre-consumption cues of predation. The contents of all feeding cups were poured into the respective predator cages $4 \mathrm{~h}$ after the start of feeding. To equalize disturbance caused by feeding, we handled cages at the beginning of feeding in $\mathrm{T} 1$ and $\mathrm{T} 2$ and added $100 \mathrm{ml}$ of tap water at the end. Once a week we rotated predators within treatments to minimize variation arising from individual predators. Starved predators in T3 and T4 were exchanged after 11 days with dragonflies that had not been fed for 3 days.

We started the experiment when $R$. temporaria tadpoles were free-swimming [stages 25, 26 (Gosner 1960)]. 
We first fed the Aeshna and added one to each cage, where appropriate. Then we added ten tadpoles to each mesocosm, one from each of the ten $R$. temporaria sibships. The resulting density of 57 tadpoles $/ \mathrm{m}^{2}$ lies well within the range found under natural conditions (Van Buskirk 2009).

We observed tadpole behaviour on days 9, 18 and 27 after starting the experiment, by visiting each mesocosm four times between 1100 and 1500 hours. On each visit, we noted the number of tadpoles that were located in the third of the mesocosm closest to the predator cage, the number of active tadpoles and the number of tadpoles visible. A tadpole was scored as being active if it was swimming or moving its tail while feeding (for similar methods, see Laurila et al. 2006; Schoeppner and Relyea 2005, 2009; Winkler and Van Buskirk 2012).

We made morphological measurements at the end of the experiment [days 28 and 29, when tadpoles were at about stage 32 (Gosner 1960)]. All tadpoles were removed, anaesthetized lightly with $0.02 \mathrm{~m} / \mathrm{m} \%$ tricaine, weighed to the nearest milligram, and photographed in lateral and ventral view with a digital camera. From the photographs we later measured head length, head depth, head width, tail length, tail fin depth and tail muscle depth (using UTHSCSA ImageTool version 3.0). These morphological measures together define the general head and tail shape of a tadpole and are sensitive to the presence of predators (Laurila et al. 2004; Teplitsky et al. 2004). The six size measurements were defined following Van Buskirk and McCollum (2000) except that tail muscle depth was measured at the location of maximum tail fin depth. We did behavioural observations and morphological measurements blindly with respect to treatment.

\section{Statistical analyses}

We tested for treatment effects on the survival, body mass, behaviour and body shape of tadpoles. Survival was the arcsine-square-root transformed proportion alive at days 28,29 . In six mesocosms there were 11 survivors, suggesting that we added more than ten tadpoles to some mesocosms when setting up the experiment. In these six cases we set survival to 1 . The error was random with respect to treatments, but our survival results must nevertheless be interpreted with some caution. Body mass was logtransformed mass on days 28, 29, after excluding seven extremely small tadpoles with mass $<300 \mathrm{mg}$ (compared with an average of $746 \mathrm{mg} \pm 131 \mathrm{SD}$ for the remaining 953 survivors). The proportions of live tadpoles close to the predator cage, active, and visible above the leaf litter were calculated assuming a linear mortality curve. The behavioural data were arcsine-square-root transformed, averaged for each date, and subjected to principal components
(PC) analysis (PCA) to produce a single component that explained $83.2 \%$ of the variance. The original measures of behaviour loaded strongly and positively on the first component (PC1; near predator cage, 0.87; activity, 0.92, visibility, 0.95). Low values corresponded to mesocosms in which tadpoles were far from the predator cage, moved little, and hid frequently under the leaf litter. This combination of behaviours is characteristic of tadpoles that are threatened by predators (Kats and Dill 1998), and is associated with elevated survival under predation threat (McCollum and Van Buskirk 1996; Laurila et al. 2006; Takahara et al. 2008).

We derived a single biologically relevant index of body shape from the six measures of head and tail. The measures were regressed against the square-root of mass, and the mesocosm-means of residuals were subjected to PCA. The first component (PC1) explained $62.5 \%$ of the variance and all original shape measures loaded strongly on it (head length, -0.86 ; head depth, 0.81, head width, 0.80 ; tail length, -0.84 ; tail fin depth, 0.85 ; tail muscle depth, 0.53). A large value of PC1 corresponded to a short tadpole with a wide and high head and deep tail fin and muscle. This combination of traits is typical of tadpoles exposed to odonate predators (Van Buskirk 2002; Relyea 2003; Laurila et al. 2004) and confers enhanced survival under predation (McCollum and Van Buskirk 1996; Teplitsky et al. 2005; Hettyey et al. 2011). PC2, mainly representing variation in tail muscle depth, responded to treatments qualitatively the same as PC1; for the sake of simplicity, we present only the results of the first component.

All responses were analysed using general linear models (GLM) with treatment and spatial block as fixed factors. The analysis of mass included the number of tadpoles as a covariate to control for variation in resource availability. The analysis of behaviour observed over three dates was a repeated-measures GLM, and when the timeby-treatment interaction was significant we fitted separate models for each date. We designed three sets of planned contrasts to address the hypotheses outlined above. A contrast among treatments T1-T5 and T9 tested the effects of cue type; that among treatments $\mathrm{T} 4$ and T6-T9 tested the effects of prey type; that among treatments $\mathrm{T} 4$, $\mathrm{T} 9$, and T10 tested the effect of prey quantity. Within the planned contrasts, we used Tukey's honest significant difference (HSD) tests for pairwise comparisons among treatments and for delineating homogeneous subsets of treatments. Appendix A gives the full list of pairwise comparisons. Multivariate analyses on the three original measures of tadpole behaviour and on the six original tadpole-shape measures yielded qualitatively very similar results (Appendices B, C). Statistical models were implemented in IBM SPSS Statistics 20. 


\section{Results}

\section{Survival and body mass}

Survival averaged 0.955 and varied significantly among blocks $\left(\mathrm{GLM} ; F_{9,81}=9.85 ; P<0.001\right)$ and treatments $\left(F_{9,81}=2.55 ; P=0.012\right)$ : it was lowest in mesocosms containing a starved dragonfly larva (mean \pm SE $0.89 \pm 0.03$ ), and was similar in all other treatments (range 0.94-1.0). Average tadpole mass also varied among blocks (GLM; $\left.F_{9,80}=8.8 ; P<0.001\right)$ and treatments $\left(\mathrm{GLM} ; F_{9,80}=5.44\right.$; $P<0.001$ ). Mass was, on average, around $770 \mathrm{mg}$ in all treatments (range 752-783 mg), except for the treatments receiving ground $R$. temporaria tadpoles $(683 \pm 16 \mathrm{mg})$ and those containing a dragonfly larva fed with $R$. temporaria tadpoles not subjected to washing after feeding $(665 \pm 25 \mathrm{mg})$. The number of tadpoles in the mesocosm at termination had a significant negative effect on body mass $\left(F_{1,80}=5.74 ; B=-0.062 ;\right.$ SE $\left.0.026 ; P=0.019\right)$.

\section{Behaviour}

Analysis of cue type (T1-T5 and T9) revealed that tadpoles were least active when exposed to predators fed on $R$. temporaria tadpoles (Fig. 1a-c). Repeated-measures analysis indicated that behaviour changed over time and varied significantly among treatments, and that the interaction between time and treatment was significant (time, $F_{2,90}=35.32 ; P<0.001$; treatment, $F_{5,45}=64.2$; $P<0.001$; time $\times$ treatment, $F_{10,90}=3.46 ; P=0.001$ ). Effects of block and its interaction with time were nonsignificant (block, $F_{9,45}=1.9 ; P=0.077$; time $\times$ block, $\left.F_{18,90}=1.54 ; P=0.094\right)$. The overall pattern of behaviour was similar on the three sampling dates. Tadpoles reacted most strongly to predators fed conspecific tadpoles (T2). The response to starved predators and homogenized conspecifics (T5) was intermediate between the control and $\mathrm{T} 2$, and differed from both (T5 vs. T1 and T2; all $P<0.002)$. Also, tadpoles exposed to homogenized tadpoles or to starved predators tended to show induced behaviour as compared to the control on all sampling dates (T3 and T4 vs. T1; 9 days after start, both $P<0.085 ; 18$ days after start, both $P<0.004 ; 27$ days after start, T3 vs. T1, $P<0.001$, T4 vs. T1, $P=1$ ). However, the responses to homogenized tadpoles or starved predators were at times weaker, and at times similar to those of tadpoles exposed to both homogenized tadpoles and starved predators (T3 and T4 vs. T5; 9 days after start, both $P<0.02 ; 18$ days after start, both $P>0.39 ; 27$ days after start, T3 vs. $5: P=1$, T4 vs. $5, P<0.001)$. Washing the predator had no effect on the behavioural response on the first two sampling dates (T2 vs. T9; both $P>0.7)$, whereas it weakened the response on the third date $(P=0.003)$.
The analysis on the behavioural effects of prey type (T4 and T6-9) revealed that tadpoles reacted more strongly to predators feeding on conspecific or phylogenetically closely related prey than to starved predators or to predators feeding on phylogenetically distantly related prey (Fig. 1d-f). Time-dependent changes in behaviour and among-treatment differences were again significant, while the interaction of time and treatment was significant as well (repeated-measures GLM; time, $F_{2,72}=55.61 ; P<0.001$; treatment, $F_{4,36}=33.08 ; P<0.001$; time $\times$ treatment, $\left.F_{8,72}=2.9 ; P=0.007\right)$. Block and its interaction with time were again non-significant (block, $F_{9,36}=1.78 ; P=0.11$; time $\times$ block, $F_{18,72}=1.41 ; P=0.16$ ). Behaviour of tadpoles exposed to predators fed with phylogenetically unrelated or distantly related prey (Chironomus or B. bufo larvae) and subsequently washed did not differ significantly at any sampling occasion from that of tadpoles exposed to starved predators (T6 and 7 vs. T4; all $P>0.16$ ), while tadpoles exposed to predators fed with conspecific prey and subsequently washed showed stronger induced changes (T4, 6 and 7 vs. T9; all $P<0.004$ ). The behaviour of tadpoles exposed to predators fed with conspecific or phylogenetically closely related prey did not differ significantly at any sampling occasion (T8 vs. 9; 9 days after start, $P=0.99$; 18 days after start, $P=0.89 ; 27$ days after start, $P=0.063$ ).

In the analysis of the effects of prey quantity, there was a strong reaction to predators fed conspecific tadpoles but no effect of the quantity of food consumed by the predators (Fig. 1g). Repeated-measures analysis revealed significant effects of treatment and time, but no interaction between them (treatment, $F_{2,18}=48.44 ; P<0.001$; time, $F_{2,36}=34.06 ; P<0.001$; treatment $\times$ time, $F_{4,36}=0.23$; $P=0.92$ ). Tadpoles exposed to predators fed conspecifics showed stronger responses than those exposed to starved predators (T4 vs. T9; $P<0.001$ ). Doubling the amount of prey did not further elevate behavioural responses (T9 vs. T10; $P=0.54)$. The effect of block and its interaction with time were both non-significant (block, $F_{9,18}=2 ; P=0.1$; block $\times$ time, $F_{18,36}=0.8 ; P=0.69$ ).

\section{Body shape}

Planned contrasts testing effects of cue type (T1-T5 and T9) revealed that homogenised tadpoles induced no change in the shape of the head and tail, and feeding predators induced a stronger change than starved predators (Fig. 2a). There was significant overall variation among treatments $\left(\mathrm{GLM} ; F_{5,45}=89.38 ; P<0.001\right)$ and blocks $\left(F_{9,45}=6.08\right.$; $P<0.001)$. Tukey's HSD post hoc tests indicated that control tadpoles did not differ significantly from tadpoles exposed solely to homogenized conspecifics $(P=0.89)$, whereas all remaining pairwise comparisons among treatments were significant (all $P<0.007$ ). 


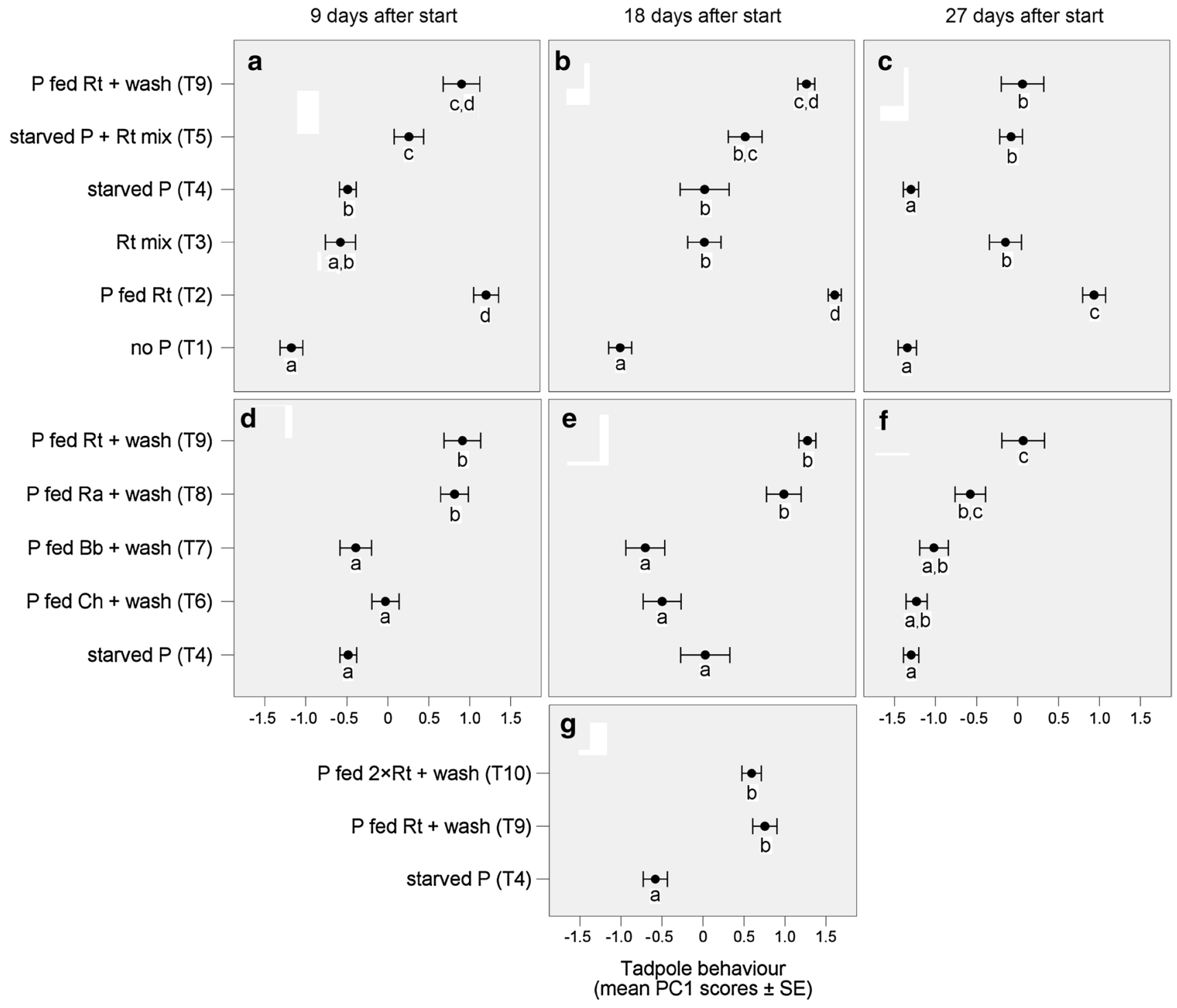

Fig. 1 Behaviour of tadpoles observed 9, 18 and 27 days after the start of the experiment ( $n=10$ in all treatments). Behaviour was calculated as principal component (PC) 1 scores of a PC analysis (PCA) on percent active, percent close to the predator cage, and percent visible. To facilitate comparison between treatment effects on behaviour and morphology, we depict component scores after multiplication by -1 , so that high values on the behaviour axis correspond to low activity, few tadpoles close to the predator cage, and few tadpoles visible. Symbols are mean \pm SE. Letters depict homogeneous subsets

Analysis on the effects of prey type (T4 and T6-T9) indicated that tadpoles had deeper tails and shorter heads when exposed to predators fed with $R$. arvalis or $R$. temporaria prey (Fig. 2b). Body shape varied among treatments and blocks (treatment, $F_{4,36}=27.73 ; P<0.001$; block, $\left.F_{9,36}=5.3 ; P<0.001\right)$. Post hoc tests showed that shape was similar in treatments where tadpoles were exposed to the smell of starved predators or predators fed with chironomid or B. bufo prey (Tukey's HSD pairwise calculated using Tukey's honest signficant difference (HSD) tests in planned comparisons on the effects of a-c cue type, d-f prey type and $\mathbf{g}$ prey quantity. The effect of prey quantity is depicted only on one panel because it was similar on all sampling occasions. $P$ Predator, no $P$ only handling the empty cage, Rt Rana temporaria tadpoles, Rt mix homogenized Rana temporaria tadpoles, Ch chironomid larvae, Bb Bufo bufo tadpoles, Ra Rana arvalis tadpoles, $2 \times$ double amount, wash predator washed three times after feeding

comparisons among T4, T6 and T7, all $P>0.18$ ) and when predators fed on $R$. arvalis or R. temporaria (T8 and T9, $P=0.83$ ). All pairwise comparisons between these two sets of treatments were significant (all $P<0.001$ ).

Doubling the quantity of prey consumed had no further impact on tadpole morphology (Fig. 2c). Comparison of $\mathrm{T} 4, \mathrm{~T} 9$ and $\mathrm{T} 10$ revealed significant variation in body shape among treatments $\left(F_{2,18}=39.3 ; P<0.001\right)$ and no block effect $\left(F_{9,18}=1.59 ; P=0.19\right)$. Post hoc tests showed that 


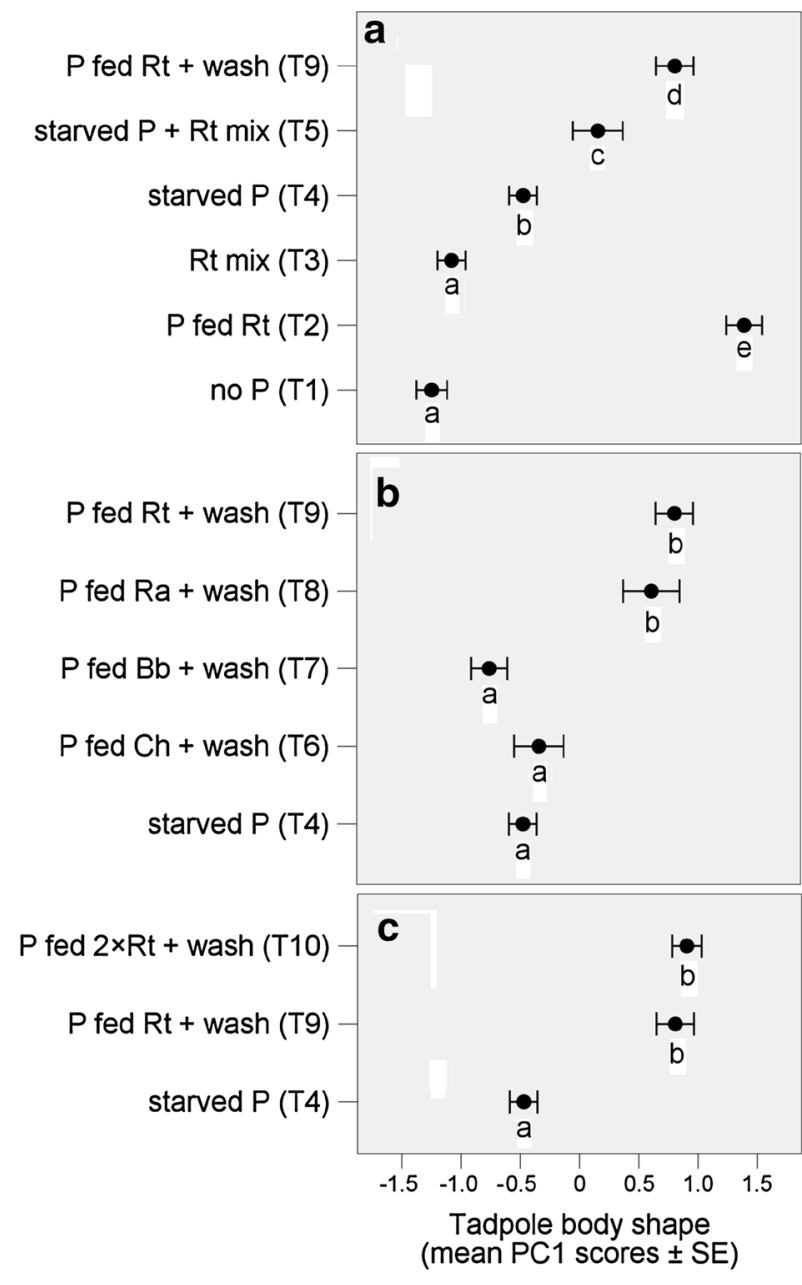

Fig. 2 Mean head and tail shape ( \pm SE) of tadpoles sampled 28 days after the start of the experiment. The figure is based on mesocosm means, consequently, sample sizes equalled ten in all treatments. Shape is a score on the first axis of a PCA on six measures of the head and tail after correcting for mass. Higher PC1 scores represent a relatively short total length with a wide and high head and deep tail fin and tail muscle, which corresponds to a tadpole reacting to the presence of an odonate predator. Letters depict homogeneous subsets calculated using Tukey's HSD tests in planned comparisons on the effects of a cue type, $\mathbf{b}$ prey type and $\mathbf{c}$ prey quantity. For abbreviations, see Fig. 1

tadpoles exposed to the smell of predators fed with conspecific tadpoles had higher values of PC1 than those exposed to starved predators (T4 vs. T9 or T10, both $P<0.001$ ), while the amount of prey did not make a significant difference (T9 vs. T10, $P=0.84$ ).

\section{Discussion}

Our study demonstrates that anuran larvae use the information encoded in both continually released predator-borne cues and digestion-released prey-borne cues to adjust antipredator responses. Both types of chemical cue proved to be necessary for the induction of the full intensity of inducible defences in $R$. temporaria tadpoles. Results for behaviour and morphology were consistent. Treatment effects on body mass were relatively weak and inconclusive, which agrees with previous reports (e.g. Relyea and Werner 2000; Van Buskirk 2001; Schoeppner and Relyea 2009). While the main results affirm those of previous studies (LaFiandra and Babbitt 2004; Schoeppner and Relyea 2005, 2009; Richardson 2006; Hettyey et al. 2010), our study supports a range of further conclusions regarding the relative importance of different types of cues, of prey type and of prey quantity.

Analysis of the types of cues that were available to focal tadpoles revealed that pre-consumption prey-borne cues in isolation induced behavioural defences but no change in body shape (T1 vs. T3). This is somewhat surprising because some predators have evolved behavioural or physiological adaptations impeding the use of predator-borne cues and digestion-released cues (Brown et al. 1995; Chivers and Smith 1998), so that pre-consumption prey-borne cues may be the only cues available for prey to adjust their defences. However, prey may reserve development of morphological changes - which take time to be expressed and can be costly-for situations in which reliable information about the predator species is available. Also, in comparison with behavioural responses to predation, morphological responses may be effective only when they are specific to the type of predator. Consequently, if only pre-consumption prey-borne cues are present and there is no information available about the predator, morphological changes may not be induced. Accordingly, studies of several other taxa agree that pre-consumption prey-borne cues in isolation elicit weak responses, frequently affecting only prey behaviour [cladocerans (Pijanowska 1997); bryozoans (Harvell 1986); snails (Turner 1996); tadpoles (Petranka and Hayes 1998); Schoeppner and Relyea (2005, 2009)].

Continually released predator-borne cues in isolation elicited both morphological and behavioural responses (T1 vs. T4). This suggests that $A$. cyanea larvae, and probably many other predators as well, release olfactory cues more or less constantly and not only when they chew or digest prey, and these can indeed be used by prey to detect predators and adjust their level of response. Evidence from previous studies is inconclusive on this point: some studies report no detectable response to predator-borne cues alone (e.g. McCollum and Leimberger 1997; Schoeppner and Relyea 2009), while others observed changes in both behaviour and morphology (e.g. Pettersson et al. 2000; Petranka and Hayes 1998; Schoeppner and Relyea 2005). This discrepancy may partly be due to differences in the traits measured, because behavioural responses may be induced by pre-consumption cues, whereas less plastic 
changes in morphology may only develop in the presence of predator-borne cues (Van Buskirk and Arioli 2002; for further references see above). Also, predator recognition may involve learning in some prey species or in the presence of some predator species [damselflies (Wisenden et al. 1997); fishes (Brown 2003); tadpoles (Gonzalo et al. 2007; Fraker 2009; Chivers and Ferrari 2013)], whereas it must be at least partly innate in many other prey or in relation to other types of predators [snails (Turner 1996); fishes (Vilhunen and Hirvonen 2003); tadpoles (Petranka and Hayes 1998; Schoeppner and Relyea 2005; Hettyey et al. 2012; this study)].

Stronger responses to continually released predatorborne cues than to pre-consumption cues may have partly resulted from our experimental methodology: predators emitting continually released cues were present almost all the time, whereas pre-consumption cues were added only once a day. However, phenotypic responses to these two types of cues in isolation tended to be weaker than when they were both available to focal tadpoles (T3, T4 vs. T5). The only comparable study found that the combined presence of pre-consumption and continually released predator-borne cues did not elicit stronger responses in tadpoles than when these cues were available in isolation (Schoeppner and Relyea 2009). This discrepancy may be attributed to methodological differences between the studies. For example, we added pre-consumption cues more frequently (seven vs. three times a week), thereby potentially causing more pronounced responses. In any case, the data indicate that detectable quantities of intact pre-consumption preyborne cues were transferred into the mesocosms in T2, T3 and $\mathrm{T} 5$, because responses to a combination of pre-consumption prey-borne cues and continually released predator-borne cues were stronger than to continually released predator-borne cues alone (T4 vs. T5).

Our results deliver several lines of evidence for the importance of digestion-released cues. First, effects of digestion-released cues added to the effects of pre-consumption cues and continually released predator-borne cues (T2 vs. T5) (for similar results see Jacobsen and Stabell 2004; Schoeppner and Relyea 2009). This also supports the hypothesis that all types of chemical cues are necessary to induce the full suite and magnitude of inducible responses in anuran larvae (Van Buskirk and Arioli 2002; LaFiandra and Babbitt 2004; Richardson 2006; Schoeppner and Relyea 2005, 2009). Second, morphological responses were stronger when continually released predator-borne cues were combined with digestion-released cues than when combined with pre-consumption cues (T5 vs. T9). There was a similar tendency in behavioural responses (also see Ferrari et al. 2007). However, the process of homogenization in a blender may not allow tadpoles to produce or release large quantities of pre-consumption cues before death (Fraker et al. 2009). Hence, the difference between T2 and T5 may be attributed to lower concentration of preconsumption cues in T5. Nonetheless, while the temporal pattern of attack-released cue synthesis is largely unknown, we are inclined to dismiss this possibility because previous studies using similar homogenization methods have induced strong behavioural responses in focal animals (Hews 1988; Schoeppner and Relyea 2005; Ferrari et al. 2008). Another explanation may be that continually released predator-borne cues are released in lower quantities by starved predators than by well-fed individuals. According to both explanations, the discrepancy between T5 and T2/T9 may simply result from differences in the concentration of predator-borne cues. Consequently, these lines of evidence for the importance of digestion-released cues require further investigation.

We found that prey type affected tadpole phenotype via digestion-released cues. The response of tadpoles exposed to cues released from phylogenetically distantly related prey via digestion by the predator was weaker than the response to digestion-released cues after consumption of phylogenetically closely related prey. Indeed, the response to predators fed with phylogenetically distant prey did not differ significantly from that of tadpoles exposed to starved predators releasing no digestion-released cues. That is, tadpoles showed no detectable difference in response to unfed and fed predators if pre-consumption cues were excluded and the predators had been fed with phylogenetically distant prey. In previous experiments, $R$. temporaria tadpoles responded similarly to predators that had been fed either conspecifics or B. bufo tadpoles (Laurila et al. 1997, 1998). However, Laurila et al. (1997, 1998) did not exclude preconsumption cues, and our data show that in the absence of these cues the response to predators consuming $B$. bufo was weaker than to predators fed conspecifics and similar to the response to starved predators. Therefore, R. temporaria tadpoles respond to attack-released cues emitted by B. bufo, but not to digestion-released cues from predators that had fed on toad larvae. This suggests that at least some types of pre-consumption cues emitted by attacked prey are conservative and universal and thus less dependent on the phylogenetic relationship between the sender and receiver (see Kiesecker et al. 1999), while the cues released through digestion contain more taxon-specific information (Ferland-Raymond et al. 2010), allowing for a relaxation of responses to predators feeding on alternative prey.

Other studies also agree that tadpoles perceive predators feeding on their conspecifics or close relatives as more dangerous than predators that have not fed recently on these types of prey (Laurila et al. 1997, 1998; Schoeppner and Relyea 2005; Richardson 2006; Fraker 2009). The usual interpretation is that by not responding to predators that are feeding on other species, prey can spare the cost of induced 
defences without experiencing elevated predation risk (Persons et al. 2001). We also find that tadpoles may use digestion-released cues to detect predators that had fed on conspecifics or close relatives but recently have not attacked further prey [also see Richardson 2006; Ferland-Raymond et al. 2010; for similar results in damselflies and fish see Mathis and Smith (1993); Chivers et al. (1996); Ferrari et al. (2007)]. Our results demonstrate especially clearly that tadpoles adjust their responses to digestion-released prey-borne cues originating from different types of prey because we held constant the quantity of digestion-released predator-borne cues [digestive fluids, gut tissue fragments, or the predator's gut microflora (Pettersson et al. 2000; Ferrari et al. 2007; Schoeppner and Relyea 2009; FerlandRaymond et al. 2010)].

Antipredator phenotypic responses were stronger to predators fed conspecifics than to starved predators, but a larger quantity of prey consumed by predators did not further enhance induced changes. That is, elevated amounts of digestion-released cues did not lead to increased responses. The absence of a graded dosage response may reflect an all-or-nothing reaction to digestion-released cues originating from conspecifics, or may indicate that our lowest treatment level was already too high to detect the graded phase. Comparison with earlier work suggests that the latter was probably not the case. McCoy et al. (2012) observed that the dose-response curve had levelled off already in response to $0.2 \mathrm{mg}$ tadpole tissue $\mathrm{L}^{-1}$ day $^{-1}$ fed to the predators, which is lower than the concentrations applied in our experiment (1.7-3.3 $\mathrm{mg} \mathrm{L}^{-1} \mathrm{day}^{-1}$ ). Nonetheless, Van Buskirk and Arioli (2002) and Hettyey et al. (2010) noted graded responses to cue concentrations as high as 5.6 and $8.8 \mathrm{mg} \mathrm{L}^{-1} \mathrm{day}^{-1}$. The latter study, like the present one, also excluded pre-consumption cues. While many studies have documented that prey animals use the concentration of pre-consumption prey-borne cues and continually released predator-borne cues to adjust their antipredatorresponses in other taxa as well [insects (Kesavaraju et al. 2007); fishes (Ferrari et al. 2005)], further research will be necessary to uncover the importance of the quantity of digestion-released cues.

In summary, our results support conclusions about the relative importance of several types of chemical cues of predation threat. Most importantly, however, we have clearly demonstrated that continually released predatorborne cues and digestion-released cues are used by tadpoles for the adjustment of antipredator defences. Using continually released predator-borne cues and digestion-released cues may enhance survival probabilities of prey by providing specific information on the type, location, abundance and recent feeding history of predators. This information could be only partially derived from pre-consumption prey-borne cues. Also, continually released predator-borne cues allow prey to detect recently relocated or unfed predators, and to recognize them as dangerous even when preconsumption prey-borne cues are absent. While these are long-standing and widely recognized theoretical considerations (e.g. Laurila et al. 1998), our study provides the most compelling and detailed empirical evidence available that continually released predator-borne cues and digestionreleased cues are used by larvae of anuran amphibians.

Author contribution statement A. H. and J. V. B. conceived and designed the study. A. H., Z. T., K. E. T. and J. G. F. collected data. A. H. and J. V. B. performed statistical analyses. A. H. and J. V. B. wrote the first draft and Z. T., K. E. T., J. G. F., and D. J. P. substantially improved the manuscript.

Acknowledgments We thank Anssi Laurila for discussions on the experimental design. The City of Vienna (MA22-231/2011) and Land Steiermark (FA13C-53S-7/2011-92) issued permissions for collecting animals, the Ethical Commission of the University of Veterinary Medicine approved the experiments in accordance with Good Scientific Practice guidelines and national legislation. The Pilisi Parkerdő Zrt. allowed us to use their forestry roads. Research was supported by the Swiss National Science Foundation (SNF, 31003A-140979), the Lendület programme of the Hungarian Academy of Sciences (MTA, LP2012-24/2012) and an FP7 Marie Curie Career Integration Grant (PCIG13-GA-2013-631722).

Conflict of interest The authors have no conflict of interest to declare.

\section{Box 1: Clarifying terminology and classifying mechanisms for chemosensory-mediated predator detection}

Many studies report the use of chemical cues to detect predators, but they employ widely different definitions and classifications of types of cues. The same terms are used sometimes as synonyms, at other times they refer to different phenomena, and definitions are often missing. For example, 'diet-released cues' can refer to those that originate from digested prey (e.g., Ferrari et al. 2007; FerlandRaymond et al. 2010), but sometimes they also include cues that are released by prey upon attack (e.g., Laurila et al. 1997; El-Balaa and Blouin-Demers 2013). Many authors use the term kairomone in reference to cues from a predator that are independent of its recent feeding history (e.g., Brönmark and Hansson 2000; Hettyey et al. 2010), others state that kairomones include digestion-released cues (e.g., Kats and Dill 1998; Schoeppner and Relyea 2005, 2009), and still others use the term kairomone whenever the receiver is a heterospecific (e.g., Chivers and Smith 1998). 
A second example of inconsistent terminology is the classification of cues as indirect or direct. Indirect cues originate from prey and have evolved to alert other prey to predation threat. They include several kinds of chemicals: general prey metabolites that are excreted actively upon stress ('no-cost disturbance signals'; Wisenden et al. 1995; Kiesecker et al. 1999), special disturbance cues that are costly to produce and are released by prey actively upon attack ('alarm pheromones'; Fraker et al. 2009), cues that are passively released from injured prey tissue ('damagereleased cues'; Chivers and Smith 1998), and cues that are released from prey by digestion ('digestion-released cues', also referred to as 'predator-labelling'; Mathis and Smith 1993; Chivers and Smith 1998; Ferrari et al. 2007). Direct cues, on the other hand, originate directly from the predator and represent the smell of the predator itself that is independent from its recent feeding history. These cues are released 'unintentionally', alerting potential prey to predation threat and lowering the predator's chance of successful attack. Direct cues include chemicals and tissue fragments that are released more or less continually from the integument of the predator ('kairomones'; Petranka and Hayes 1998; Brönmark and Hansson 2000), saliva released during capture and consumption of prey (we know of no study demonstrating this), and digestive body fluids of the predator, tissue fragments of the predators' digestive tract and samples of the predators' gut flora released during excretion ('digestion-released cues'; Mathis and Smith 1993; Ferrari et al. 2007). As can be seen from the above list, excrements of predators may contain both indirect and direct cues. Furthermore, kairomones may be released not only continually from the integument of predators, but also during defecation (fractions of 'digestion-released cues'). This further confuses functional and physiological/mechanistic classification. Finally, some of the current nomenclature is based on functionality and some on the timing of release, while cue origin is only implicitly understood.

To improve clarity, help avoid misunderstandings and facilitate comparability of results, we propose a new terminology for the cues involved in chemosensory-mediated predator detection. We suggest using a binomial nomenclature and classification based on the timing of cue release (stress-, attack-, capture-, digestion- or continually-released cues) in combination with cue origin (prey-borne versus predator-borne cues) (Table 1).

\section{References}

Brönmark C, Hansson L-A (2000) Chemical communication in aquatic systems. Oikos 88:103-109

Brown GE (2003) Learning about danger: chemical alarm cues and local risk assessment. Fish Fish 4:227-234
Brown GE, Chivers DP, Smith RJF (1995) Localized defecation by pike: a response to labelling by cyprinid alarm pheromone? Behav Ecol Sociobiol 36:105-110

Chivers DP, Ferrari MCO (2013) Tadpole antipredator responses change over time: what is the role of learning and generalization? Behav Ecol 24:1114-1121

Chivers DP, Smith RJF (1998) Chemical alarm signalling in aquatic predator-prey systems: a review and prospectus. Ecoscience $5: 338-352$

Chivers DP, Wisenden BD, Smith RJF (1996) Damselfly larvae learn to recognize predators from chemical cues in the predator's diet. Anim Behav 52:315-320

DeWitt TJ, Sih A, Wilson DS (1998) Costs and limits of phenotypic plasticity. Trends Ecol Evol 13:77-81

El-Balaa R, Blouin-Demers G (2013) Does exposure to cues of fish predators fed different diets affect morphology and performance of Northern Leopard Frog (Lithobates pipiens) larvae? Can J Zool 91:203-211

Ferland-Raymond B, March RE, Metcalfe CD, Murray DL (2010) Prey detection of aquatic predators: assessing the identity of chemical cues eliciting prey behavioral plasticity. Biochem Syst Ecol 38:169-177

Ferrari MCO, Trowell JJ, Brown GE, Chivers DP (2005) The role of learning in the development of threat-sensitive predator avoidance by fathead minnows. Anim Behav 70:777-784

Ferrari MCO, Brown MR, Pollock MS, Chivers DP (2007) The paradox of risk assessment: comparing responses of fathead minnows to capture-released and diet-released alarm cues from two different predators. Chemoecology 17:157-161

Ferrari MCO, Messier F, Chivers DP (2008) Degradation of chemical alarm cues under natural conditions: risk assessment by larval woodfrogs. Chemoecology 17:263-266

Fraker ME (2008) The dynamics of predation risk assessment: responses of anuran larvae to chemical cues of predators. J Anim Ecol 77:638-645

Fraker ME (2009) Predation risk assessment through chemical cues produced by multiple prey. Behav Ecol Sociobiol 63:1397-1402

Fraker ME, Hu F, Cuddapah V, McCollum SA, Relyea RA, Hempel J, Denver RJ (2009) Characterization of an alarm pheromone secreted by amphibian tadpoles that induces behavioral inhibition and suppression of the neuroendocrine stress axis. Horm Behav 55:520-529

Gonzalo A, Lopez P, Martin J (2007) Iberian green frog tadpoles may learn to recognize novel predators from chemical alarm cues of conspecifics. Anim Behav 74:447-453

Gosner KL (1960) A simplified table for staging anuran embryos and larvae with notes on their identification. Herpetologica $16: 183-190$

Harvell CD (1986) The ecology and evolution of inducible defences in a marine bryozoan: cues, costs and consequences. Am Nat 128:810-823

Harvell CD (1990) The ecology and evolution of inducible defences. Q Rev Biol 65:323-340

Hettyey A, Zsarnóczai S, Vincze K, Hoi H, Laurila A (2010) Interactions between the information content of different chemical cues affect induced defences in tadpoles. Oikos 119:1814-1822

Hettyey A, Vincze K, Zsarnóczai S, Hoi H, Laurila A (2011) Costs and benefits of defenses induced by predators differing in dangerousness. J Evol Biol 24:1007-1019

Hettyey A, Rölli F, Thürlimann N, Zürcher A-C, Van Buskirk J (2012) Visual cues contribute to predator detection in anuran larvae. Biol J Linn Soc 106:820-827

Hews DK (1988) Alarm response in larval western toads, Bufo boreas-release of larval chemicals by a natural predator and its effect on predator capture efficiency. Anim Behav 36:125-133 
Jacobsen HP, Stabell OB (2004) Antipredator behaviour mediated by chemical cues: the role of conspecific alarm signaling and predator labeling in the avoidance response of a marine gastropod. Oikos 104:43-50

Kats LB, Dill LM (1998) The scent of death: chemosensory assessment of predation risk by prey animals. Ecoscience 5:361-394

Kesavaraju B, Damal K, Juiano SA (2007) Threat-sensitive behavioral responses to concentrations of water-borne cues from predation. Ethology 113:119-206

Kiesecker JM, Chivers DP, Marco A, Quilchano C, Anderson MT, Blaustein AR (1999) Identification of a disturbance signal in larval red-legged frogs, Rana aurora. Anim Behav 57:1295-1300

Kishida O, Nishimura K (2005) Multiple inducible defences against multiple predators in the anuran tadpole, Rana pirica. Evol Ecol Res 7:619-631

LaFiandra EM, Babbitt KJ (2004) Predator induced phenotypic plasticity in the pinewoods tree frog, Hyla femoralis: necessary cues and the cost of development. Oecologia 138:350-359

Laurila A, Kujasalo J, Ranta E (1997) Different antipredator behaviour in two anuran tadpoles: effects of predator diet. Behav Ecol Sociobiol 40:329-336

Laurila A, Kujasalo J, Ranta E (1998) Predator-induced changes in life history in two anuran tadpoles: effects of predator diet. Oikos 83:307-317

Laurila A, Järvi-Laturi M, Pakkasmaa S, Merilä J (2004) Temporal variation in predation risk: stage dependency, graded responses and fitness costs in tadpole antipredator defences. Oikos 107:90-99

Laurila A, Pakkasmaa S, Merilä J (2006) Population divergence in growth rate and antipredator defences in Rana arvalis. Oecologia 147:585-595

Mathis A, Smith RJF (1993) Fathead minnows, Pimephales promelas, learn to recognize northern pike, Esox lucius, as predators on the basis of chemical stimuli from minnows in the pike's diet. Anim Behav 46:645-656

McCollum SA, Leimberger JD (1997) Predator-induced morphological changes in an amphibian: predation by dragonflies affects tadpole shape and color. Oecologia 109:615-621

McCollum SA, Van Buskirk J (1996) Costs and benefits of a predator-induced polyphenism in the gray treefrog Hyla chrysoscelis. Evolution 50:583-593

McCoy MW, Touchon JC, Landberg T, Warkentin KM, Vonesh JR (2012) Prey responses to predator chemical cues: Disentangling the importance of the number and biomass of prey consumed. PLoS One 7:e47495

Moran NA (1992) The evolutionary maintenance of alternative phenotypes. Am Nat 139:971-989

Peacor SD (2006) Behavioural response of bullfrog tadpoles to chemical cues of predation risk are affected by cue age and water source. Hydrobiologia 573:39-44

Persons MH, Walker SE, Rypstra AL, Marshall SD (2001) Wolf spider predator avoidance tactics and survival in the presence of diet-associated predator cues (Araneae: Lycosidae). Anim Behav 61:43-51

Petranka J, Hayes L (1998) Chemically mediated avoidance of a predatory odonate (Anax junius) by American toad (Bufo americanus) and wood frog (Rana sylvatica) tadpoles. Behav Ecol Sociobiol 42:263-271

Pettersson LB, Nilsson PA, Brönmark C (2000) Predator recognition and defence strategies in crucian carp, Carassius carassius. Oikos 88:200-212

Pijanowska J (1997) Alarm signals in Daphnia? Oecologia 112:12-16

Relyea RA (2003) How prey respond to combined predators: a review and an empirical test. Ecology 84:1827-1839
Relyea RA, Werner EE (2000) Morphological plasticity in four larval anurans distributed along an environmental gradient. Copeia 2000:178-190

Richardson JL (2006) Novel features of an inducible defense system in larval tree frogs (Hyla chrysoscelis). Ecology 87:780-787

Schoeppner NM, Relyea RA (2005) Damage, digestion, and defence: the roles of alarm cues and kairomones for inducing prey defences. Ecol Lett 8:505-512

Schoeppner NM, Relyea RA (2008) Detecting small environmental differences: risk-response curves for predator-induced behavior and morphology. Oecologia 154:743-754

Schoeppner NM, Relyea RA (2009) Interpreting the smells of predation: how alarm cues and kairomones induce different prey defences. Funct Ecol 23:1114-1121

Stauffer HP, Semlitsch RD (1993) Effects of visual, chemical and tactile cues of fish on the behavioral responses of tadpoles. Anim Behav 46:355-364

Takahara T, Kohmatsu Y, Maruyama A, Yamaoka R (2008) Benefit of suites of defensive behavior induced by predator chemical cues on anuran tadpoles, Hyla japonica. Behav Ecol Sociobiol 63:235-240

Teplitsky C, Laurila A (2007) Flexible defense strategies: competition modifies investment in behavioral vs. morphological defenses. Ecology 88:1641-1646

Teplitsky C, Plenet S, Joly P (2004) Hierarchical responses of tadpoles to multiple predators. Ecology 85:2888-2894

Teplitsky C, Plenet S, Léna JP, Mermet N, Malet E, Joly P (2005) Escape behaviour and ultimate causes of specific induced defences in an anuran tadpole. J Evol Biol 18:180-190

Tollrian R, Harvell CD (1999) The ecology and evolution of inducible defences. Princeton University Press, Princeton

Turner AM (1996) Freshwater snails alter habitat use in response to predation. Anim Behav 51:747-756

Van Buskirk J (2001) Specific induced responses to different predator species in anuran larvae. J Evol Biol 14:482-489

Van Buskirk J (2002) A comparative test of the adaptive plasticity hypothesis: relationships between habitat and phenotype in anuran larvae. Am Nat 160:87-102

Van Buskirk J (2009) Natural variation in morphology of larval amphibians: phenotypic plasticity in nature? Ecol Monogr 79:681-705

Van Buskirk J, Arioli M (2002) Dosage response of an induced defense: how sensitive are tadpoles to predation risk? Ecology 83:1580-1585

Van Buskirk J, McCollum SA (2000) Influence of tail shape on tadpole swimming performance. J Exp Biol 203:2149-2158

Van Buskirk J, Krugel A, Kunz J, Miss F, Stamm A (2014) The rate of degradation of chemical cues indicating predation risk: an experiment and review. Ethology 120:942-949

Vilhunen S, Hirvonen H (2003) Innate antipredator responses of Arctic charr (Salvelinus alpinus) depend on predator species and their diet. Behav Ecol Sociobiol 55:1-10

Wilson RS, Kraft PG, Van Damme R (2005) Predator-specific changes in the morphology and swimming performance of larval Rana lessonae. Funct Ecol 19:238-244

Winkler JD, Van Buskirk J (2012) Influence of experimental venue on phenotype: multiple traits reveal multiple answers. Funct Ecol 26:513-521

Wisenden BD, Chivers DP, Smith RJF (1995) Early warning in the predation sequence: a disturbance pheromone in Iowa darters (Etheostoma exile). J Chem Ecol 21:1469-1480

Wisenden BD, Chivers DP, Smith RJF (1997) Learned recognition of predation risk by Enallagma damselfly larvae (Odonata, Zygoptera) on the basis of chemical cues. J Chem Ecol 23:137-151 